\title{
Influência da época de colheita e do armazenamento em atmosfera controlada na qualidade da maçã 'Braeburn'(1)
}

\author{
Auri Brackmann ${ }^{(2)}$, Cristiano André Steffens ${ }^{(3)}$ e Alessandro Jaquiel Waclawovsky(2)
}

\begin{abstract}
Resumo - Foi avaliado o efeito da época de colheita e das condições de armazenamento em atmosfera controlada sobre a firmeza da polpa, acidez titulável, ${ }^{\circ}$ Brix e, principalmente, sobre a ocorrência de degenerescência da polpa em maçãs (Malus domestica cv. Braeburn). Os tratamentos consistiram na combinação da data de colheita (27/3/1997 e 9/4/1997) com condição de armazenamento (temperatura de $0,5^{\circ} \mathrm{C}$ com: $1 \mathrm{kPa}$ de $\mathrm{O}_{2} / 4,0 \mathrm{kPa}$ de $\mathrm{CO}_{2} ; 1 \mathrm{kPa}$ de $\mathrm{O}_{2} / 3,0 \mathrm{kPa}$ de $\mathrm{CO}_{2} ; 1 \mathrm{kPa}$ de $\mathrm{O}_{2} / 2,0 \mathrm{kPa}$ de $\mathrm{CO}_{2} ; 21 \mathrm{kPa}$ de $\mathrm{O}_{2} / 0,0 \mathrm{kPa}$ de $\mathrm{CO}_{2}$, e temperatura de $-0,5^{\circ} \mathrm{C}$ com: $1 \mathrm{kPa}$ de $\mathrm{O}_{2} / 3,0 \mathrm{kPa}$ de $\mathrm{CO}_{2} ; 1 \mathrm{kPa}$ de $\mathrm{O}_{2} / 2,0 \mathrm{kPa}$ de $\mathrm{CO}_{2}$ ). Após oito meses de armazenamento, não foi observada suscetibilidade da maçã $\mathrm{cv}$. Braeburn à baixa temperatura de armazenamento $\left(-0,5^{\circ} \mathrm{C}\right)$ e os frutos armazenados em ambiente refrigerado apresentaram baixa qualidade para o consumo. As condições de atmosfera controlada de $1 \mathrm{kPa}$ de $\mathrm{O}_{2}$ associadas com 2 e $3 \mathrm{kPa}$ de $\mathrm{CO}_{2}$ e a $-0,5^{\circ} \mathrm{C}$ apresentaram menor incidência de podridões, rachaduras e degenerescência senescente. Os frutos colhidos tardiamente, em 9/4/1997, apresentaram maior incidência de podridões, polpa farinhenta, degenerescência com cortiça e rachaduras. Nos parâmetros firmeza da polpa, acidez titulável e teor de sólidos solúveis totais não se observaram diferenças entre as condições de atmosfera controlada, após sete dias de exposição à temperatura de $25^{\circ} \mathrm{C}$.
\end{abstract}

Termos para indexação: Malus domestica, pós-colheita, distúrbios fisiológicos, degradação.

Influence of harvest maturity and controlled atmosphere conditions on the quality of 'Braeburn' apple

\begin{abstract}
This work was carried out to evaluate the effect of harvest maturity and controlled atmosphere on the quality and internal breakdown incidence in apples (Malus domestica) cv. Braeburn. The treatments consisted in the combination of harvest maturity (March 27, 1997 and April 9, 1997) with storage conditions $\left(0.5^{\circ} \mathrm{C}\right.$ with: $1 \mathrm{kPa} \mathrm{O}_{2} / 4 \mathrm{kPa} \mathrm{CO} 2,1 \mathrm{kPa} \mathrm{O} / 3 \mathrm{kPa} \mathrm{CO}_{2} ; 1 \mathrm{kPa} \mathrm{O} / 2 \mathrm{kPa} \mathrm{CO}_{2}$; $21 \mathrm{kPa} \mathrm{O}_{2} / 0 \mathrm{kPa} \mathrm{CO}$-cold storage and; $-0.5^{\circ} \mathrm{C}$ with: $1 \mathrm{kPa} \mathrm{O}_{2} / 3 \mathrm{kPa} \mathrm{CO}_{2} ; 1 \mathrm{kPa} \mathrm{O}_{2} / 2 \mathrm{kPa} \mathrm{CO}_{2}$ ). After eight months of storage, there was no low temperature injury on fruits stored at $-0.5^{\circ} \mathrm{C}$, and the cold storage fruits presented low quality for consumption. Under controlled atmosphere conditions of $1 \mathrm{kPa} \mathrm{O}$ combined with 2 and $3 \mathrm{kPa} \mathrm{CO}_{2}$ in $-0.5^{\circ} \mathrm{C}$, the fruits presented lower rot, fruit cracking and internal breakdown incidence. Late harvested fruits (April 9, 1997) presented higher rot, mealy pulps, cork breakdown and craked fruits incidence. For flesh firmness, titratable acidity and TSS there was no significant difference among controlled atmosphere conditions after seven days in shelf life.
\end{abstract}

Index terms: Malus domestica, postharvest, physiological disorders, degradation.

\section{Introdução}

A maçã (Malus domestica Borkh.) cv. Braeburn vem se destacando internacionalmente pela sua excelente qualidade; no mercado Europeu, atinge pre-

(1) Aceito para publicação em 14 de maio de 2001 .

(2) Universidade Federal de Santa Maria (UFSM), Centro de Ciências Rurais (CCR), Dep. de Fitotecnia, RS-509, km 9, CEP 97105-900 Santa Maria, RS. E-mail: brackman@ccr.ufsm.br

(3) UFSM. Bolsista Pibic/CNPq. E-mail: postharvest@bol.com.br ços superiores aos de outras cultivares tradicionais. Entretanto, essa cultivar tem apresentado problemas pós-colheita, como a manifestação de degenerescência interna durante o armazenamento, que culminam com o escurecimento interno da polpa (Lau, 1997).

Alguns autores (Reyes Fernandez, 1994; Burmeister \& Roughan, 1997; Watkins et al., 1997) afirmaram que as causas do escurecimento da polpa estão diretamente relacionadas com fatores pré-colheita, como clima, pomar, data da colheita e fatores pós-colheita. Segundo Argenta et al. (1994), maçãs 
colhidas tardiamente podem apresentar pingo-de-mel e, conseqüentemente, apresentar-se mais suscetíveis à ocorrência de degenerescência da polpa durante o armazenamento.

Segundo Hardenburg et al. (1986), a temperatura de armazenamento, na maioria das cultivares, é de $-1^{\circ} \mathrm{C}$ a $0^{\circ} \mathrm{C}$. Johnson et al. (1989) constataram que a temperatura de armazenamento varia em virtude da suscetibilidade dos frutos aos danos causados por baixas temperaturas. Conforme Reyes Fernandez (1994), o armazenamento refrigerado (AR), na temperatura de $0^{\circ} \mathrm{C}$, permitiu conservar a qualidade dos frutos por um período de até seis meses. Lau (1997), por sua vez, não verificou efeito da temperatura sobre a incidência de degenerescência da polpa na maçã cv. Braeburn, mas afirma que frutos armazenados em atmosfera controlada (AC) apresentaram-se mais firmes e com maior teor de acidez titulável, em relação aos frutos armazenados em AR. Para Banks et al. (1997) e Watkins et al. (1997), a condição de armazenamento ideal para maçã cv. Braeburn foi $2 \mathrm{kPa}$ de $\mathrm{O}_{2}$ e $1 \mathrm{kPa}$ de $\mathrm{CO}_{2}$. Meheriuk (1993) recomendou que a atmosfera da câmara contenha $1 \mathrm{a} 2 \mathrm{kPa} \mathrm{O}_{2}$ e 1 a $2 \mathrm{kPa}$ de $\mathrm{CO}_{2}$. Banks et al. (1997) afirmaram que a adição de $\mathrm{CO}_{2}$ na câmara faz-se necessária para minimizar perda de firmeza, mas a pressão parcial de $\mathrm{CO}_{2}$ deve ser menor ou, no máximo, igual à pressão parcial de $\mathrm{O}_{2}$.

No entanto, Lau (1997) e Kupferman (1997) relatam elevada incidência de degenerescência da polpa em frutos armazenados em $\mathrm{AC}$, o que atribuíram à baixa difusividade da epiderme aos gases $\left(\mathrm{O}_{2} / \mathrm{CO}_{2}\right)$ e ao adensamento das células que prejudicam as trocas gasosas. Lau (1997) citou que a temperatura do ar durante o desenvolvimento dos frutos tem influência nesses fatores. Devido a esse fato, pode existir diferença entre locais e anos pesquisados quanto à suscetibilidade dessa cultivar ao $\mathrm{CO}_{2}$. Na Nova Zelândia, a cv. Braeburn pode ser armazenada com $3 \mathrm{kPa}$ de $\mathrm{O}_{2}$ e $1 \mathrm{kPa}$ de $\mathrm{CO}_{2}$ e temperatura de $0,5^{\circ} \mathrm{C}$, enquanto nos Estados Unidos, é recomendado $2 \mathrm{kPa}$ de $\mathrm{O}_{2}$ com o $\mathrm{CO}_{2}$ abaixo de $0,5 \mathrm{kPa}$ e temperatura de $1,5^{\circ} \mathrm{C}$ (Kupferman, 1997). No Brasil, Brackmann \& Waclawovsky (2000) recomendaram $1 \mathrm{kPa}$ de $\mathrm{O}_{2} \mathrm{e}$ $3 \mathrm{kPa}$ de $\mathrm{CO}_{2}$ na temperatura de $0^{\circ} \mathrm{C}$ e afirmaram que essa cultivar apresentou elevada incidência de degenerescência quando foi armazenada em AR. Este é, provavelmente, o único trabalho desenvolvido com essa cultivar nas condições edafoclimáticas brasileiras.

O presente trabalho foi realizado com o objetivo de avaliar o efeito da época da colheita e das condições de armazenamento em atmosfera controlada, sobre as características físico-químicas e sobre a ocorrência da degenerescência da polpa em maçãs cv. Braeburn.

\section{Material e Métodos}

O experimento foi realizado no Núcleo de Pesquisa em Pós-colheita (NPP), do Departamento de Fitotecnia da Universidade Federal de Santa Maria, RS, durante o ano de 1997. Foram utilizadas maçãs da cultivar Braeburn, procedentes de um pomar comercial da região de Vacaria, RS (latitude: $28^{\circ} 33^{\prime}$; altitude: $955 \mathrm{~m}$ ). Na colheita do dia 27/3/1997, os frutos apresentavam índice de iodo-amido de 5,6 , firmeza de polpa de $88,83 \mathrm{~N}$, teor de SST de $12,5^{\circ}$ Brix e acidez titulável de $6,76 \mathrm{cmol} \mathrm{L}^{-1}$. Os frutos, colhidos no dia 9/4/1997, apresentavam índice de iodo-amido de 4,3, firmeza de polpa de $85,79 \mathrm{~N}$, teor de SST de $12,8^{\circ}$ Brix, e acidez titulável de $6,34 \mathrm{cmol} \mathrm{L}^{-1}$. Estes frutos apresentaram elevada incidência $(66 \%)$ de pingo-de-mel. Após as colheitas, os frutos foram transportados, em temperatura ambiente, para Santa Maria, distante $500 \mathrm{~km}$ do pomar onde se efetuaram as colheitas. No NPP foram selecionados, eliminado-se os que apresentavam lesões ou estavam fora das especificações em termos de tamanho e, em seguida, foram homogeneizadas as amostras experimentais.

No armazenamento refrigerado, as minicâmaras, com volume de $232 \mathrm{~L}$, permaneceram semi-fechadas para a manutenção de altos níveis de umidade relativa e baixos de $\mathrm{CO}_{2}$. Já no armazenamento em AC, foram fechadas hermeticamente para que as pressões parciais de gases pudessem ser controladas.

O delineamento experimental utilizado foi o inteiramente casualizado com três repetições, sendo a unidade experimental composta por 60 frutos. Os tratamentos avaliados consistiram na combinação da data de colheita (27/3/1997 e 9/4/1997) com condições de armazenamento (temperatura de $0,5^{\circ} \mathrm{C}$ com: $1 \mathrm{kPa}$ de $\mathrm{O}_{2} / 4 \mathrm{kPa}$ de $\mathrm{CO}_{2}$; $1 \mathrm{kPa}$ de $\mathrm{O}_{2} / 3 \mathrm{kPa}$ de $\mathrm{CO}_{2} ; 1 \mathrm{kPa}$ de $\mathrm{O}_{2} / 2 \mathrm{kPa}$ de $\mathrm{CO}_{2}$; $21 \mathrm{kPa}$ de $\mathrm{O}_{2} / 0 \mathrm{kPa}$ de $\mathrm{CO}_{2}(\mathrm{AR})$, e temperatura de $-0,5^{\circ} \mathrm{C}$ com: $1 \mathrm{kPa}$ de $\mathrm{O}_{2} / 3 \mathrm{kPa}$ de $\mathrm{CO}_{2} ; 1 \mathrm{kPa}$ de $\mathrm{O}_{2} / 2 \mathrm{kPa}$ de $\mathrm{CO}_{2}$ ). A temperatura de armazenamento foi medida na polpa do fruto e teve uma oscilação de $\pm 0,2^{\circ} \mathrm{C}$. A instalação da atmosfera nas minicâmaras de $\mathrm{AC}$ ocorreu 24 horas após as colheitas. As pressões parciais de gases foram obtidas 
por diluição do $\mathrm{O}_{2}$ com injeção de $\mathrm{N}_{2}$ e posterior injeção de $\mathrm{CO}_{2}$, comprimido em cilindros de alta pressão, até atingir os níveis preestabelecidos. A manutenção da pressão parcial desejada dos gases, nas diferentes minicâmaras, foi feita por análise diária com o uso de analisadores de $\mathrm{O}_{2} \mathrm{e}$ $\mathrm{CO}_{2}$ e posterior correção das pressões parciais, as quais variavam de acordo com a respiração dos frutos. O gás carbônico foi absorvido por meio da circulação do gás das minicâmaras por um absorvedor de $\mathrm{CO}_{2}$, que continha uma solução de $\mathrm{KOH}$ (40\%). Para compensar o oxigênio consumido pela respiração dos frutos, foi injetado ar atmosférico nas minicâmaras. As pressões parciais dos gases foram expressas em $\mathrm{kPa}$, considerando-se $1,0 \%$ aproximadamente igual a $1 \mathrm{kPa}$. A umidade relativa, mensurada com auxílio de um psicrômetro, permaneceu em torno de $96 \%$.

$\mathrm{Na}$ instalação do experimento, fez-se o teste de iodoamido e determinou-se a firmeza da polpa, o teor de sólidos solúveis totais (SST), a acidez titulável e a incidência do pingo-de-mel. Após oito meses de armazenamento, os 60 frutos de cada repetição foram divididos em duas subamostras de 30 frutos, sendo a primeira analisada após a retirada dos frutos das condições de armazenamento (saída da câmara) e, a segunda, após sete dias de climatização à temperatura de $25^{\circ} \mathrm{C}$, visando simular o período de beneficiamento e comercialização.

$\mathrm{O}$ índice iodo-amido foi determinado pelo método de Streif (1984), que utiliza uma tabela de fotografias, com índice 01 para o teor máximo de amido e índice 10 para o amido totalmente hidrolisado. A firmeza de polpa dos frutos foi avaliada utilizando-se um penetrômetro motorizado com ponteira de $11 \mathrm{~mm}$ de diâmetro e o teor de SST, por refratometria. Na determinação da acidez titulável, foram coletados $10 \mathrm{~mL}$ de suco de uma amostra de 20 frutos de cada repetição, que, após diluído em $100 \mathrm{~mL}$ de água, foi titulado com $\mathrm{NaOH} 0,1 \mathrm{~N}$ até $\mathrm{pH} 8,1$. Foram determinados também a ocorrência de podridões, podridãocarpelar, degenerescência senescente, degenerescência com cortiça, rachaduras e escaldadura. Estas determinações foram feitas pela contagem dos frutos que apresentavam sintomas característicos desses danos. Frutos com lesões maiores que $5 \mathrm{~mm}$, com características de ataque de patógenos, foram considerados podres. A podridãocarpelar caracteriza-se pela presença de micélio de patógenos na região das lojas carpelares e, num estádio mais avançado, pelo mesocarpo colonizado por fungos. A degenerescência senescente se caracteriza por regiões da polpa com escurecimento e com aspecto umedecido. A degenerescência com cortiça se caracteriza por pequenas lesões de aspecto corticento e seco e, às vezes, com pequenas cavernas próximas ao miolo do fruto.

Os dados foram submetidos à análise de variância e as médias comparadas pelo teste de Duncan a $5 \%$ de probabilidade. Valores expressos em porcentagem foram transformados pela fórmula arc sen $(\mathrm{x} / 100)^{0,5}$ antes da análise de variância.

\section{Resultados e Discussão}

Após oito meses de armazenamento, na saída da câmara, os resultados da análise da variância revelaram significância da interação entre os fatores data de colheita e condição de armazenamento para acidez titulável, podridão-carpelar e degenerescência senescente e, após sete dias, para firmeza de polpa, podridão, degenerescência senescente e degenerescência com cortiça.

A data da colheita dos frutos não influenciou na manutenção da firmeza de polpa e acidez titulável, tanto na saída da câmara como após sete dias de climatização dos frutos a $25^{\circ} \mathrm{C}$ (Tabela 1). Este resultado contraria o comportamento observado em outras cultivares de maçã (Argenta et al., 1994).

O uso da atmosfera controlada proporcionou frutos com maior firmeza de polpa e acidez titulável nas duas avaliações realizadas, na saída da câmara e após sete dias. Lau (1997) e Brackmann \& Waclawovsky (2000) também obtiveram maior firmeza de polpa e acidez titulável em maçãs cv. Braeburn armazenadas em AC. Watkins (1998) e Brackmann \& Waclawovsky (2000) explicaram esse efeito da AC sobre a firmeza da polpa e acidez dos frutos, como resultado da redução da produção e ação do etileno e redução da respiração e da degradação das pectinas da parede celular. Entretanto, nesse trabalho não foi verificado efeito do aumento de 2 para $4 \mathrm{k}$ Pa de $\mathrm{CO}_{2}$, na atmosfera da câmara e na manutenção da firmeza de polpa. Brackmann et al. (1996) obtiveram, na maçã 'Golden Delicious', melhor manutenção da firmeza da polpa com o aumento da pressão parcial de $\mathrm{CO}_{2}$.

Pesq. agropec. bras., Brasília, v. 37, n. 3, p. 295-301, mar. 2002 
Tabela 1. Características físico-químicas de maçãs cv. Braeburn, colhidas em duas datas, após oito meses de armazenamento refrigerado e em atmosfera controlada e sete dias de exposição à temperatura de $25^{\circ} \mathrm{C}$. Santa Maria, RS. $1997^{(1)}$.

\begin{tabular}{|c|c|c|c|c|c|c|c|c|c|c|}
\hline \multirow{2}{*}{$\begin{array}{c}\text { Tratamento } \\
\left(\mathrm{kPa} \mathrm{O}_{2} / \mathrm{kPa} \mathrm{CO}_{2}\right)\end{array}$} & \multirow{2}{*}{$\begin{array}{l}\text { Temperatura } \\
\left({ }^{\circ} \mathrm{C}\right)\end{array}$} & \multicolumn{3}{|c|}{ Firmeza da polpa (N) } & \multicolumn{3}{|c|}{ Acidez titulável $\left(\mathrm{cmol} \mathrm{L}^{-1}\right)$} & \multicolumn{3}{|c|}{ SST ( ${ }^{\circ}$ Brix) } \\
\hline & & $27 / 3 / 97$ & 9/4/97 & Média & $27 / 3 / 97$ & 9/4/97 & Média & 27/3/97 & 9/4/97 & Média \\
\hline & & \multicolumn{9}{|c|}{ Na colheita } \\
\hline & - & 83,83 & 85,79 & & 6,79 & 6,34 & & 12,50 & 12,80 & \\
\hline & & \multicolumn{9}{|c|}{ Após oito meses de armazenamento } \\
\hline $1,0 / 4,0$ & 0,5 & 70,34 & 67,60 & $68,97 \mathrm{a}$ & $5,62 \mathrm{aA}$ & $5,61 \mathrm{abA}$ & 5,62 & 14,02 & 14,00 & $14,08 \mathrm{ab}$ \\
\hline $1,0 / 3,0$ & 0,5 & 72,73 & 62,47 & $67,60 \mathrm{a}$ & $5,48 \mathrm{aA}$ & $5,63 \mathrm{abA}$ & 5,56 & 14,17 & 13,83 & $14,00 \mathrm{ab}$ \\
\hline $1,0 / 2,0$ & 0,5 & 70,42 & 64,26 & $67,34 \mathrm{a}$ & $5,79 \mathrm{aA}$ & $5,53 \mathrm{bA}$ & 5,66 & 14,43 & 13,87 & $14,15 \mathrm{a}$ \\
\hline $1,0 / 3,0$ & $-0,5$ & 70,62 & 74,31 & $72,47 a$ & $5,73 \mathrm{aA}$ & $5,72 \mathrm{bA}$ & 5,73 & 13,90 & 13,70 & $13,80 \mathrm{ab}$ \\
\hline $1,0 / 2,0$ & $-0,5$ & 72,70 & 69,64 & $71,17 \mathrm{a}$ & $5,60 \mathrm{aA}$ & $5,93 \mathrm{aA}$ & 5,77 & 13,87 & 13,63 & $13,75 b$ \\
\hline $21,0 / 0,0$ & 0,5 & 48,05 & 49,20 & $48,62 \mathrm{~b}$ & $3,64 \mathrm{bB}$ & $4,37 \mathrm{cA}$ & 4,00 & 13,17 & 13,50 & $13,33 \mathrm{c}$ \\
\hline \multirow[t]{2}{*}{ Média } & & $67,48 \mathrm{~A}$ & $64,58 \mathrm{~A}$ & & 5,31 & 5,46 & & $13,93 \mathrm{~A}$ & $13,76 \mathrm{~A}$ & \\
\hline & & \multicolumn{9}{|c|}{ Após sete dias a $25^{\circ} \mathrm{C}$} \\
\hline $1,0 / 4,0$ & 0,5 & $68,49 \mathrm{aA}$ & $66,26 \mathrm{aA}$ & 67,37 & 5,06 & 5,24 & $5,15 \mathrm{a}$ & 14,20 & 14,20 & $14,20 \mathrm{a}$ \\
\hline $1,0 / 3,0$ & 0,5 & $74,77 \mathrm{aA}$ & $52,74 \mathrm{bB}$ & 63,76 & 5,14 & 5,15 & $5,15 \mathrm{a}$ & 14,37 & 13,90 & $14,13 \mathrm{a}$ \\
\hline $1,0 / 2,0$ & 0,5 & $70,58 \mathrm{aA}$ & $66,78 \mathrm{aA}$ & 68,68 & 5,31 & 5,80 & $5,56 \mathrm{a}$ & 14,57 & 14,00 & $14,28 \mathrm{a}$ \\
\hline $1,0 / 3,0$ & $-0,5$ & $74,15 \mathrm{aA}$ & $75,09 \mathrm{aA}$ & 74,62 & 5,12 & 5,38 & $5,25 \mathrm{a}$ & 14,37 & 14,20 & $14,28 \mathrm{a}$ \\
\hline $1,0 / 2,0$ & $-0,5$ & $76,49 \mathrm{aA}$ & $69,84 \mathrm{aA}$ & 73,17 & 5,53 & 5,34 & $5,43 \mathrm{a}$ & 14,30 & 14,07 & $14,18 \mathrm{a}$ \\
\hline $21,0 / 0,0$ & 0,5 & $43,59 \mathrm{bA}$ & $39,87 \mathrm{cA}$ & 41,73 & 2,84 & 3,12 & $2,98 b$ & 14,30 & 13,77 & $14,03 \mathrm{a}$ \\
\hline Média & & 68,01 & 61,76 & & $4,84 \mathrm{~A}$ & $5,01 \mathrm{~A}$ & & $14,35 \mathrm{~A}$ & $14,02 \mathrm{~B}$ & \\
\hline
\end{tabular}

${ }^{(1)}$ Médias seguidas por letras diferentes, maiúscula na vertical e minúscula na horizontal, diferem entre si pelo teste de Duncan a $5 \%$ de probabilidade. 
Os níveis de SST eram menores nos frutos armazenados em AR na saída da câmara. Após sete dias a $25^{\circ} \mathrm{C}$, não se observou diferença estatística entre as condições de armazenamento. Brackmann \& Waclawovsky (2000) também observaram menores valores de SST nos frutos armazenados em AR, mas só após sete dias de exposição dos frutos a $25^{\circ} \mathrm{C}$. Este resultado é proveniente da elevada atividade metabólica durante o armazenamento em AR com a conseqüente degradação dos açúcares. Quanto à data da colheita dos frutos, o efeito foi verificado somente após sete dias, sendo que os frutos colhidos precocemente, no dia 27/3/1997, apresentaram os maiores valores de SST. Argenta et al. (1994) também afirmam que maçãs colhidas tardiamente podem apresentar menor conservação dos açúcares.

Quanto à ocorrência de podridões, na saída da câmara, os frutos armazenados em $1 \mathrm{kPa}$ de $\mathrm{O}_{2}$ e $4 \mathrm{kPa}$ de $\mathrm{CO}_{2}$ e à temperatura de $0,5^{\circ} \mathrm{C}$ apresentaram maior incidência de podridões (Tabela 2). Este resultado contraria os obtidos por Brackmann \& Saquet (1995), em que as maçãs cv. Gala revelaram redução no ataque de patógenos com o acréscimo na pressão parcial de $\mathrm{CO}_{2}$. Após sete dias, os frutos colhidos no dia 27/3/1997 e armazenados em $\mathrm{AC}$ e à temperatura de $-0,5^{\circ} \mathrm{C}$, juntamente com os frutos armazenados em $1 \mathrm{kPa}$ de $\mathrm{O}_{2}$ e $3 \mathrm{kPa}$ de $\mathrm{CO}_{2}$ e temperatura de $-0,5^{\circ} \mathrm{C}$, apresentaram menor incidência de podridões.

A incidência de frutos com podridão-carpelar, na saída da câmara, ocorreu somente nos frutos colhidos em 9/4/1997, armazenados em 1 kPa de $\mathrm{O}_{2}$ e $3 \mathrm{kPa}$ de $\mathrm{CO}_{2}$ e à temperatura de $0,5^{\circ} \mathrm{C}$ (Tabela 2). Após sete dias, as incidências de podridão-carpelar e de frutos com escaldadura não foram significativas e nem associadas a nenhuma das condições de armazenamento.

Houve ocorrência de frutos com rachaduras somente na saída da câmara, com maior magnitude nos frutos colhidos tardiamente, em 9/4/1997, o que se deveu à avançada maturação.

A incidência de degenerescência senescente, na saída da câmara, foi mais elevada nos frutos colhidos em 9/4/1997, armazenados em AC e na maior temperatura de armazenamento $\left(0,5^{\circ} \mathrm{C}\right)$ e nas pressões parciais de $\mathrm{CO}_{2}$ mais baixas (2 e $3 \mathrm{kPa}$ ) (Tabela 3). Este resultado, quanto à data de colheita, está de acordo com Argenta et al. (1994), Burmeister \& Roughan (1997) e Elgar et al. (1997), que afirmaram que maçãs colhidas tardiamente apresentaram-se mais suscetíveis à ocorrência de escurecimento da polpa. Após sete dias, os frutos, colhidos em 27/3/1997, apresentaram elevada incidência desse distúrbio somente no armazenamento em AR, o que pode ser decorrente da avançada maturação nessa condição de armazenamento. No entanto, Elgar et al. (1997) e Watkins et al. (1997) consideraram que a degenerescência da maçã cv. Braeburn está associada com o armazenamento em AC. Brackmann \& Waclawovsky (2000) também verificaram maior ocorrência desse distúrbio em frutos armazenados em AR. Já os frutos, colhidos tardiamente, em 9/4/1997, apresentaram-se mais suscetíveis à incidência desse distúrbio, com exceção dos frutos armazenados em alto $\mathrm{CO}_{2}(4 \mathrm{kPa})$.

A ocorrência de degenerescência com cortiça, tanto na saída da câmara como após sete dias a $25^{\circ} \mathrm{C}$, foi menor nos frutos armazenados em AR (Tabela 3 ). Após sete dias a $25^{\circ} \mathrm{C}$, apenas os frutos que foram colhidos em 9/4/1997 e armazenados em AC apresentaram incidência do distúrbio. Esse resultado, assim como a ocorrência de degenerescência senescente, está de acordo com a hipótese de que a ocorrência de degenerescências aumenta com o atraso na data da colheita (Burmeister \& Roughan, 1997; Elgar et al., 1997). Entretanto, resultados obtidos por Brackmann \& Waclawovsky (2000) contrariam, em parte, os resultados obtidos no presente trabalho, pois verificaram maior ocorrência de degenerescência com cortiça nos frutos conservados em AR.

A incidência de frutos com polpa farinhenta foi mais elevada no armazenamento refrigerado, em ambas as avaliações. Brackmann \& Saquet (1995), em 'Gala', também verificaram maior incidência desse distúrbio em condições de armazenamento refrigerado, o que proporcionou acelerado amadurecimento dos frutos, tornando-os farinhentos. Após sete dias a $25^{\circ} \mathrm{C}$, também verificou-se que os frutos colhidos tardiamente, em 9/4/1997, apresentaram maior incidência do distúrbio. 
Tabela 2. Ocorrência de podridões e distúrbios fisiológicos em maçãs cv. Braeburn, colhidas em duas datas, após oito meses de armazenamento refrigerado e em atmosfera controlada e mais sete dias de exposição à temperatura de $25^{\circ} \mathrm{C}$. Santa Maria, RS, $1997^{(1)}$

\begin{tabular}{|c|c|c|c|c|c|c|c|c|c|c|}
\hline \multirow{2}{*}{$\begin{array}{c}\text { Tratamento } \\
\left(\mathrm{kPa} \mathrm{O}_{2} / \mathrm{kPa} \mathrm{CO}_{2}\right)\end{array}$} & \multirow{2}{*}{$\begin{array}{c}\text { Temperatura } \\
\left({ }^{\circ} \mathrm{C}\right)\end{array}$} & \multicolumn{3}{|c|}{ Podridão (\%) } & \multicolumn{3}{|c|}{ Podridão-carpelar (\%) } & \multicolumn{3}{|c|}{ Rachaduras (\%) } \\
\hline & & $27 / 3 / 97$ & 9/4/97 & Média & $27 / 3 / 97$ & 9/4/97 & Média & $27 / 3 / 97$ & $9 / 4 / 97$ & Média \\
\hline & & \multicolumn{9}{|c|}{ Após oito meses de armazenamento } \\
\hline $1,0 / 4,0$ & 0,5 & 10,7 & 26,7 & $18,7 \mathrm{a}$ & $0,0 \mathrm{aA}$ & $0,0 \mathrm{bA}$ & 0,0 & 1,3 & 11,7 & $6,5 \mathrm{a}$ \\
\hline $1,0 / 3,0$ & 0,5 & 10,7 & 17,4 & $14,1 \mathrm{ab}$ & $0,0 \mathrm{aB}$ & $18,2 \mathrm{aA}$ & 9,1 & 1,3 & 10,5 & $5,9 \mathrm{a}$ \\
\hline $1,0 / 2,0$ & 0,5 & 4,0 & 27,0 & $15,5 \mathrm{ab}$ & $0,0 \mathrm{aA}$ & $0,0 \mathrm{bA}$ & 0,0 & 0,0 & 5,0 & $2,5 \mathrm{ab}$ \\
\hline $1,0 / 3,0$ & $-0,5$ & 14,7 & 10,0 & $12,3 a b$ & $0,0 \mathrm{aA}$ & $0,0 \mathrm{bA}$ & 0,0 & 2,7 & 0,0 & $1,3 \mathrm{ab}$ \\
\hline $1,0 / 2,0$ & $-0,5$ & 5,3 & 1,8 & $3,5 \mathrm{~b}$ & $0,0 \mathrm{aA}$ & $0,0 \mathrm{bA}$ & 0,0 & 0,0 & 0,0 & $0,0 \mathrm{~b}$ \\
\hline $21,0 / 0,0$ & 0,5 & 5,0 & 9,6 & $7,3 \mathrm{ab}$ & $0,0 \mathrm{aA}$ & $0,0 \mathrm{bA}$ & 0,0 & 1,7 & 3,3 & $2,5 \mathrm{ab}$ \\
\hline \multirow{2}{*}{ Média } & & $8,4 \mathrm{~A}$ & $15,4 \mathrm{~A}$ & & 0,0 & 3,0 & & $1,2 \mathrm{~B}$ & $5,1 \mathrm{~A}$ & \\
\hline & & \multicolumn{9}{|c|}{ Após sete dias a $25^{\circ} \mathrm{C}$} \\
\hline $1,0 / 4,0$ & 0,5 & $16,2 \mathrm{bcB}$ & $65,7 \mathrm{aA}$ & 41,0 & 0,0 & 0,0 & $0,0 \mathrm{a}$ & 0,0 & 0,0 & 0,0 \\
\hline $1,0 / 3,0$ & 0,5 & $22,2 \mathrm{bB}$ & $61,7 \mathrm{aA}$ & 42,0 & 0,0 & 8,9 & $4,4 \mathrm{a}$ & 0,0 & 0,0 & 0,0 \\
\hline $1,0 / 2,0$ & 0,5 & $27,6 \mathrm{bB}$ & $51,5 \mathrm{aA}$ & 39,5 & 6,1 & 3,7 & $4,9 \mathrm{a}$ & 0,0 & 0,0 & 0,0 \\
\hline $1,0 / 3,0$ & $-0,5$ & $11,3 \mathrm{bcA}$ & $17,4 \mathrm{bA}$ & 14,4 & 0,0 & 0,0 & $0,0 \mathrm{a}$ & 0,0 & 0,0 & 0,0 \\
\hline $1,0 / 2,0$ & $-0,5$ & $8,3 \mathrm{cB}$ & $61,8 \mathrm{aA}$ & 35,1 & 0,0 & 3,3 & $1,7 \mathrm{a}$ & 0,0 & 0,0 & 0,0 \\
\hline $21,0 / 0,0$ & 0,5 & $58,1 \mathrm{aA}$ & $53,3 \mathrm{aA}$ & 55,7 & 0,0 & 0,0 & $0,0 \mathrm{a}$ & 0,0 & 0,0 & 0,0 \\
\hline Média & & 24,0 & 51,9 & & $1,0 \mathrm{~A}$ & $2,6 \mathrm{~A}$ & & 0,0 & 0,0 & \\
\hline
\end{tabular}

${ }^{(1)}$ Médias seguidas por letras diferentes, maiúscula na vertical e minúscula na horizontal, diferem entre si pelo teste de Duncan a $5 \%$ de probabilidade.

Tabela 3. Ocorrência de distúrbios fisiológicos em maçãs cv. Braeburn, colhidas em duas datas, após oito meses de armazenamento refrigerado e em atmosfer controlada e mais sete dias de exposição dos frutos a $25^{\circ} \mathrm{C}$. Santa Maria, RS, $1997^{(1)}$.

\begin{tabular}{|c|c|c|c|c|c|c|c|c|c|c|c|c|c|}
\hline \multirow{2}{*}{$\begin{array}{l}\text { Tratamento } \\
\left(\mathrm{kPa} \mathrm{O}_{2} / \mathrm{kPa} \mathrm{CO}_{2}\right)\end{array}$} & \multirow{2}{*}{$\begin{array}{c}\text { Temperatura } \\
\left({ }^{\circ} \mathrm{C}\right)\end{array}$} & \multicolumn{3}{|c|}{ Degenerescência senescente (\%) } & \multicolumn{3}{|c|}{ Degenerescência com cortiça (\%) } & \multicolumn{3}{|c|}{ Escaldadura (\%) } & \multicolumn{3}{|c|}{ Polpa farinhenta (\%) } \\
\hline & & $27 / 3$ & $9 / 4$ & Média & $27 / 3$ & $9 / 4$ & Média & $27 / 3$ & $9 / 4$ & Média & $27 / 3$ & $9 / 4$ & Média \\
\hline & & \multicolumn{12}{|c|}{ Após oito meses de armazenamento } \\
\hline $1,0 / 4,0$ & 0,5 & $5,6 \mathrm{aA}$ & $13,7 \mathrm{bcA}$ & 9,6 & 6,1 & 13,1 & $9,6 a b$ & 0,0 & 0,0 & $0,0 \mathrm{a}$ & 0,0 & 4,8 & $2,4 \mathrm{~b}$ \\
\hline $1,0 / 3,0$ & 0,5 & $0,0 \mathrm{aB}$ & $32,6 \mathrm{aA}$ & 16,3 & 2,8 & 0,0 & $1,4 \mathrm{c}$ & 0,0 & 0,0 & $0,0 \mathrm{a}$ & 2,8 & 3,7 & $3,2 \mathrm{~b}$ \\
\hline $1,0 / 2,0$ & 0,5 & $0,0 \mathrm{aB}$ & $23,6 \mathrm{abA}$ & 11,8 & 0,0 & 0,0 & $0,0 \mathrm{c}$ & 0,0 & 0,0 & $0,0 \mathrm{a}$ & 0,0 & 3,7 & $1,8 \mathrm{~b}$ \\
\hline $1,0 / 3,0$ & $-0,5$ & $3,3 \mathrm{aA}$ & $4,2 \mathrm{cA}$ & 3,8 & 2,8 & 7,4 & $5,1 \mathrm{bc}$ & 0,0 & 0,0 & $0,0 \mathrm{a}$ & 0,0 & 0,0 & $0,0 \mathrm{~b}$ \\
\hline $1,0 / 2,0$ & $-0,5$ & $0,0 \mathrm{aA}$ & $11,6 \mathrm{bcA}$ & 5,8 & 5,6 & 25,5 & $15,5 \mathrm{a}$ & 0,0 & 0,0 & $0,0 \mathrm{a}$ & 0,0 & 3,0 & $1,5 \mathrm{~b}$ \\
\hline $21,0 / 0,0$ & 0,5 & $0,0 \mathrm{aA}$ & $0,0 \mathrm{cA}$ & 0,0 & 0,0 & 0,0 & $0,0 \mathrm{c}$ & 0,0 & 1,5 & $0,8 \mathrm{a}$ & 47,8 & 39,8 & $43,8 \mathrm{a}$ \\
\hline \multirow[t]{2}{*}{ Média } & & 1,5 & 14,3 & & $2,9 \mathrm{~A}$ & $7,7 \mathrm{~A}$ & & $0,0 \mathrm{~A}$ & $0,2 \mathrm{~A}$ & & $8,4 \mathrm{~A}$ & $9,2 \mathrm{~A}$ & \\
\hline & & \multicolumn{12}{|c|}{ Após sete dias a $25^{\circ} \mathrm{C}$} \\
\hline $1,0 / 4,0$ & 0,5 & $8,1 \mathrm{bA}$ & $0,0 \mathrm{bA}$ & 4,1 & $0,0 \mathrm{aB}$ & $9,8 \mathrm{bcA}$ & 4,9 & 0,0 & 0,0 & 0,0 & 2,6 & 6,3 & $4,4 \mathrm{~b}$ \\
\hline $1,0 / 3,0$ & 0,5 & $2,8 \mathrm{bB}$ & $36,9 \mathrm{aA}$ & 19,9 & $0,0 \mathrm{aB}$ & $20,3 \mathrm{aA}$ & 10,2 & 0,0 & 0,0 & 0,0 & 0,0 & 21,9 & $11,0 \mathrm{~b}$ \\
\hline $1,0 / 2,0$ & 0,5 & $2,6 \mathrm{bA}$ & $15,3 \mathrm{abA}$ & 8,9 & $0,0 \mathrm{aB}$ & $7,9 \mathrm{bcA}$ & 3,9 & 0,0 & 0,0 & 0,0 & 5,6 & 7,9 & $6,7 \mathrm{~b}$ \\
\hline $1,0 / 3,0$ & $-0,5$ & $7,4 \mathrm{bA}$ & $21,7 \mathrm{aA}$ & 14,5 & $0,0 \mathrm{aB}$ & $4,2 \mathrm{cdA}$ & 2,1 & 0,0 & 0,0 & 0,0 & 3,7 & 6,7 & $5,2 \mathrm{~b}$ \\
\hline $1,0 / 2,0$ & $-0,5$ & $8,3 \mathrm{bA}$ & $18,2 \mathrm{aA}$ & 13,2 & $0,0 \mathrm{aB}$ & $14,1 \mathrm{abA}$ & 7,0 & 0,0 & 0,0 & 0,0 & 5,1 & 10,4 & $7,8 \mathrm{~b}$ \\
\hline $21,0 / 0,0$ & 0,5 & $62,5 \mathrm{aA}$ & $39,3 \mathrm{aA}$ & 50,9 & $0,0 \mathrm{aA}$ & $0,0 \mathrm{dA}$ & 0,0 & 0,0 & 0,0 & 0,0 & 79,2 & 100,0 & $89,6 \mathrm{a}$ \\
\hline Média & & 15,3 & 21,9 & & 0,0 & 9,4 & & 0,0 & 0,0 & & $16,0 \mathrm{~B}$ & $25,4 \mathrm{~A}$ & \\
\hline
\end{tabular}

${ }^{(1)}$ Médias seguidas por letras diferentes, maiúscula na vertical e minúscula na horizontal, diferem entre si pelo teste de Duncan a $5 \%$ de probabilidade. 


\section{Conclusão}

1. Maçãs 'Braeburn' colhidas tardiamente apresentam maior suscetibilidade à incidência de podridões e distúrbios fisiológicos.

2. A atmosfera controlada mantém melhor qualidade dos frutos em relação ao armazenamento refrigerado.

3. A melhor condição para o armazenamento da cultivar 'Braeburn' em atmosfera controlada é $1 \mathrm{kPa}$ de $\mathrm{O}_{2}$ combinado com $3 \mathrm{kPa}$ de $\mathrm{CO}_{2}$, na temperatura de $-0,5^{\circ} \mathrm{C}$

4. A maçã 'Braeburn' cultivada nas condições climáticas brasileiras não é tão suscetível à degenerescência no armazenamento em atmosfera controlada com alto $\mathrm{CO}_{2}$ quanto maçãs produzidas em países mais frios.

\section{Referências}

ARGENTA, L. C.; BRACKMAN, A.; MONDARDO, M. Maturação na colheita e qualidade de maçãs 'Gala' após a armazenagem. Revista Brasileira de Fisiologia Vegetal, São Carlos, v. 6, n. 2, p. 135-140, 1994.

BANKS, N. H.; JEFFERY, P. B.; MacKAY, B. R. Responses of colour change and softening to $\mathrm{O}_{2}$ and $\mathrm{CO}_{2}$ in 'Braeburn' apples. In: INTERNATIONAL CONTROLLED ATMOSPHERE RESEARCH CONFERENCE, 7., 1997, Davis. Proceedings... Davis: University of California, 1997. v. 2, p. 36-41.

BRACKMANN, A.; SAQUET, A. A. Armazenamento de maçã cv. Gala em atmosfera controlada. Revista Brasileira de Agrociência, Pelotas, v. 1, n. 2, p. 55-60, 1995.

BRACKMANN, A.; WACLAWOVSKY, A. J. Conservação da maçã (Malus domestica Borkh.) cv. Braeburn. Ciência Rural, Santa Maria, v. 30, n. 2, p. 229-234, 2000.

BRACKMANN, A.; GONÇALVES, E. D.; SAQUET, A. A. Efeito de tratamentos com altas concentrações de $\mathrm{CO}_{2}$ sobre a qualidade de maçãs 'Golden Delicious' armazenadas em atmosfera controlada. Ciência Rural, Santa Maria, v. 26, n. 2, p. 181-184, 1996.

BURMEISTER, D. M.; ROUGHAN, S. Physiological and biochemical basis for the Braeburn browning disorder (BBD). In.: INTERNATIONAL CONTROLLED ATMOSPHERE RESEARCH CONFERENCE, 7., 1997. Davis. Proceedings... Davis: University of California, 1997. v. 2, p. 126-131.

ELGAR, H. J.; BURMEISTER, D. M.; WATKINS, C. B $\mathrm{CO}_{2}$ and $\mathrm{O}_{2}$ effects on a browning disorder in 'Braeburn' apple. In.: INTERNATIONAL CONTROLLED ATMOSPHERE RESEARCH CONFERENCE, 7., 1997. Davis. Proceedings... Davis: University of California, 1997. v. 2, p. 198-203.

HARDENBURG, R. E.; WATADA, A. E.; WANG, C. Y. The commercial storage of fruits, vegetables, and florist and nursery stocks. Washington: Usda, 1986. 136 p. (Agriculture Handbook, 66).

JOHNSON, D. S.; BAILEY, T. C.; RIDOUT, M. S. Meteorological nutritional data used to predict low temperature breakdown in stored Bramley's seedling apples. Acta Horticulturae, Leuven, n. 258, p. 455-464, 1989.

KUPFERMAN, E. Controlled atmosphere storage of apples. In: INTERNATIONAL CONTROLLED ATMOSPHERE RESEARCH CONFERENCE, 7., 1997, Davis. Proceedings... Davis: University of California, 1997. v. 2, p. 1-30.

LAU, O. L. Influence of climate, harvest maturity, waxing, $\mathrm{O}_{2}$ and $\mathrm{CO}_{2}$ on browning disorders of 'Braeburn' apples. In: INTERNATIONAL CONTROLLED ATMOSPHERE RESEARCH CONFERENCE, 1997, Davis. Proceedings... Davis: University of California, 1997. v. 2, p. 132-137.

MEHERIUK, M. CA storage conditions for apples, pears, and nashi. In: INTERNATIONAL CONTROLLED ATMOSPHERE RESEARCH CONFERENCE, 6., 1993, Ithaca. Proceedings... Ithaca: Northeast Region Agricultural Engineering Service, 1993. v. 2, p. 819-858.

REYES FERNANDEZ, M. S. Manejo de cosecha y poscosecha de manzanas 'Braeburn'. Revista Frutícola, Curico, v. 15, n. 3, p. 95-101, 1994.

STREIF, J. Jod-Stärke-Test zur Beurteilung der Fruchtreife bei Äpfeln. Obst und Garten, Stuttgart, v. 8, p. 382-384, 1984.

WATKINS, C. B. Mechanisms for beneficial and deleterious responses of fruits to elevated carbon dioxide concentrations during storage. Acta Horticulturae, Leuven, n. 464 , p. $345-350,1998$.

WATKINS, C. B.; BURMEISTER, D. M.; ELGAR, H. J.; FU, W. L. A comparison of two carbon dioxide-related injuries of apple fruit. In: INTERNATIONAL CONTROLLED ATMOSPHERE RESEARCH CONFERENCE, 7., 1997, Davis. Proceedings... Davis: University of California, 1997. v. 2, p. 119-124. 